\title{
Social Skill and Social Cognition in Adolescents at Genetic Risk for Psychosis
}

\author{
Clare M. Gibson ${ }^{a}{ }^{\star}$, David L. Penn ${ }^{a}$, Mitchell J. Prinstein $^{\mathrm{a}}$, Diana O. Perkins ${ }^{\mathrm{b}}$, and Aysenil \\ Belger ${ }^{a, b}$ \\ aDepartment of Psychology, University of North Carolina at Chapel Hill \\ bDepartment of Psychiatry, University of North Carolina at Chapel Hill
}

\begin{abstract}
Adolescents at genetic high risk (GHR) for schizophrenia have shown social skill impairments and there is some evidence to suggest they have Theory of Mind (ToM) deficits; however no research has used a standardized, performance-based behavioral measure to assess social functioning in this population nor evaluated ToM with a well-validated measure. We evaluated the psychometric properties of a new, theoretically-derived assessment of social functioning in GHR adolescents: the "High-Risk Social Challenge" task (HiSoC). The second aim was to explore whether GHR adolescents would show social skill and ToM deficits as compared to a non-psychiatric control (NPC) group. The present study evaluated social functioning with the HiSoC and ToM with the Eyes Test in 23 GHR adolescents and 31 NPCs. The HiSoC demonstrated high levels of reliability and validity. The GHR adolescents showed social skills impairments, but not ToM deficits. The results suggest that the HiSoC is a potentially useful new measure of social functioning in GHR adolescents. Furthermore, the findings add to the current body of literature that indicates that social skill impairments are related to schizophrenia vulnerability.
\end{abstract}

\section{Keywords}

schizophrenia; vulnerability; social skill; theory of mind

\section{Introduction}

\subsection{Social functioning in genetic high risk adolescents}

Adolescents with a first-degree relative with schizophrenia have social impairments which are related to increased vulnerability for later disease onset (Tarbox \& Pogue-Geile, 2008). Relative to non-psychiatric controls (NPCs), adolescents at genetic high risk (GHR) for schizophrenia have poorer peer relationships (Glatt et al. 2006), fewer hobbies and interests (Dworkin et al. 1994) and lower social adjustment (Hans et al. 2000). These findings indicate that impaired social functioning in GHR adolescents is a marker of disease vulnerability (Stone et al. 2005).

\footnotetext{
(C) 2010 Elsevier B.V. All rights reserved.

*Corresponding Author: Clare Marks Gibson, M.A., Department of Psychology, CB \#3270, University of North Carolina at Chapel Hill, Chapel Hill, NC 27599-3270, cmarks@unc.edu .

This is a PDF file of an unedited manuscript that has been accepted for publication. As a service to our customers we are providing this early version of the manuscript. The manuscript will undergo copyediting, typesetting, and review of the resulting proof before it is published in its final citable form. Please note that during the production process errors may be discovered which could affect the content, and all legal disclaimers that apply to the journal pertain.
} 
Our current understanding of social functioning in GHR adolescents is based primarily on clinical interviews or participant self-reports (e.g., Dworkin et al. 1994; Stone et al. 2005). These measures are problematic in that responses to the questions may be confounded by social desirability. In addition, GHR adolescents may lack insight into their own subtle social deficits (Mueser \& Bellack, 1998) and thus more objective measures are needed to elucidate the role of social skill deficits as a vulnerability marker.

Indeed, objective performance-based measures have been used to examine social skills deficits in individuals with schizophrenia (for a review, see Mueser \& Bellack, 1998). Performance-based measures have also been used to study social functioning retrospectively in children who later developed schizophrenia (Litter \& Walker, 1993; Walker et al. 1993) and prospectively in GHR pre-adolescents and young adolescents (Schiffman et al. 2004b). Schiffman and colleagues (2004b) videotaped participants aged 11 to 13 years old while they were eating lunch with another study participant. The results showed that preadolescents and young adolescents who later developed schizophrenia were rated as less sociable than those not at risk for schizophrenia. However, Schiffman and colleagues examined a limited range of behavior and did not assess other behaviors found in the highrisk literature (e.g., social anxiety; Calkins et al. 2004). Also, the interaction was not standardized, therefore making it difficult to compare social behavior across participants. This suggests that a psychometrically sound means of assessing social behavior in GHR adolescents is lacking in the field.

\subsection{Theory of Mind in genetic high risk individuals}

An additional way of understanding social functioning in GHR adolescents is to examine social cognition. Social cognition is of great interest in schizophrenia due to its consistent association with social functioning (Couture et al. 2006). One component of social cognition, Theory of Mind (ToM), is the ability to infer another person's mental state and is critical to effective social functioning (Corcoran, 2001; Roncone et al. 2002).

ToM impairments are well documented in individuals with schizophrenia (for reviews, see Brune, 2005; Sprong et al. 2007), but this area of research has received less attention in GHR adolescents (Sprong et al. 2007). The few studies that have been conducted using adult populations show conflicting results. Some have found no difference in ToM between GHR adults and controls (e.g., Irani et al. 2006; Kelemen et al. 2004) while others have found GHR adults are impaired relative to NPCs (e.g., Janssen et al. 2003; Marjoram et al. 2006).

In the only study that investigated ToM in GHR adolescents, Schiffman and colleagues (2004a) had pre-adolescents and young adolescents at GHR for schizophrenia complete the Feffer's Role-Taking Task (RTT), a measure of perspective taking. Those who later developed schizophrenia spectrum disorders performed worse on the FTT than those who did not later develop a schizophrenia spectrum disorder. This provides preliminary support for ToM as a vulnerability marker for schizophrenia.

\subsection{The present study: aims and hypotheses}

The primary purpose of the present study was to examine social skills and ToM in adolescents at GHR for schizophrenia. The High-Risk Social Challenge (HiSoC) task was developed to assess social functioning in GHR adolescents. The HiSoC is a videotaped speech challenge task similar to those implemented in the anxiety literature (e.g., Gramer \& Saria, 2007). A coding scheme, derived from the GHR literature, was created to assess performance on the HiSoC. We hypothesized that GHR adolescents would be more impaired in social skill and ToM relative to non-clinical control participants. 


\section{Methods}

\subsection{Participants}

Twenty-three GHR adolescents were recruited from community referrals, the University of North Carolina (UNC) Chapel Hill inpatient unit, flyers in UNC hospitals and from other high risk studies conducted at UNC. Thirty-one non-psychiatric controls (NPCs) were recruited through flyers in the community and mass emails to the UNC community. The participants were being recruited as part of the Mapping Cortical Circuit Maturation in High Risk Adolescents study at the Conte Center at UNC.

Males and females between the ages of 9 and 18 were eligible to participate. Inclusion criteria for the GHR adolescents included having a first-degree relative with a Schizophrenia or Schizoaffective Disorder diagnosis according to DSM-IV-TR criteria using the Family Interview for Genetic Studies (FIGS; Maxwell, 1996). The affected first-degree relatives' diagnoses were confirmed with the Structured Clinical Interview for DSM-IV-TR Axis I Disorders (SCID-I; First et al. 2002). Study exclusion criteria for both the GHR and NPC groups included: Past or current DSM-IV-TR Axis I Psychotic Disorder or Bipolar Affective Disorder, as assessed with the Kiddie Schedule for Affective Disorders and SchizophreniaPresent and Lifetime Version (K-SADS-PL; Kaufman et al. 1997), past or current prodromal classification, diagnosis of a major central nervous system disorder, impaired intellectual functioning (WAIS-III/WISC-III IQ < 65), current treatment with antipsychotics or a past history of more than 12 weeks lifetime cumulative treatment with an antipsychotic. The NPC group had the additional exclusion criteria of no first-degree relative with a psychotic disorder using DSM-IV-TR diagnostic criteria.

\subsection{Measures}

2.2.1 Social functioning measures-Social functioning was assessed with the HighRisk Social Challenge task (HiSoC). This is a test of social skills where participants are asked to perform a mock 45 -second audition for a new reality show and are instructed to demonstrate why they should be chosen for the show.

The HiSoC was coded with a rating scheme derived from the GHR literature (e.g., Kendler et al. 1995; Docherty et al. 2004) and developed specifically for this task. The manual comprises 16 items rated on a five point Likert scale (higher scores indicating better social skills). See Table 1 for a listing of all the items on the HiSoC. For example, the verbal expression item assesses language and tone of the speech. Raters evaluate verbal expressiveness, such as tonal variation, inflection, and elaboration on speech content. The appearance item evaluates behavior that seems unusual and is not relevant to the speech task, such as excessively gesturing with hands so that it becomes distracting to the listener. The odd speech item assesses both content and style; for instance, if speech is lacking logical associations between sentences and topics are not relevant to the study activity. The items on the HiSoC were constructed to yield four theoretically derived dimensions: Affect, Odd Behavior and Language, Communication, and Social-Interpersonal. A summary item assessing the rater's overall impression of the participant is included in the $16 \mathrm{HiSoC}$ items. For more information on the item coding, please contact the lead author (CMG) for a copy of the coding manual.

The HiSoC rating scheme was primarily modeled after the Interpersonal Measure of Schizoidia and Schizotypy (Kosson et al. 1999). The IM-SS assesses schizophreniaspectrum behaviors and was chosen as a referent measure due to the association between at risk status and schizotypal traits (Kendler et al. 1995). However, the IM-SS was not used in the present study as it was developed for a standardized interaction during a semi-structured 
interview or a role-play lasting for a significant amount of time. Furthermore, the items on the HiSoC's Communication factor were not adapted from the IM-SS. These items were derived from the literature documenting communication deficits in GHR individuals (e.g., Docherty et al. 2004).

The HiSoC was videotaped and rated independently by a total of three trained undergraduate raters, with two raters scoring each speech sample. The raters were blind to group status and trained to high levels of inter-rater reliability on the hypothesized factors (i.e., ICCs > .80).

Social functioning was also assessed with the Global Functioning-Social Scale (GF-S; Auther et al. 2006) by trained research clinicians. This scale yields a single global social functioning score between 0 and 10, with higher scores indicating better social functioning.

2.2.2 Social cognition measure-ToM was assessed with the Reading the Mind in the Eyes Test-- Revised Version (Eyes Test). A trained research assistant administered both the adult (Baron-Cohen et al. 2001a) and child's (Baron-Cohen et al. 2001b) versions of the Eyes Test. The adult's version consists of 36 photographs and the child's version consists of 28 photographs. Participants are asked to guess the mental state using the four choice words. Participants under 16-years-old were administered the child's version, participants older than 16-years-old were administered the adult's version, and 16-year-olds were administered both versions. For the six participants that received both versions of the Eyes Test (i.e., the 16-year-olds), only the child's version was used in the analyses. This version was chosen to diminish the possibility of practice effects as it was administered first in the Eyes Test sequence. Performance on the Eyes Test is measured by the number of faces correctly discriminated.

2.2.3 Clinical measure-The Structured Interview for Prodromal Syndromes (SIPS; McGlashan et al. 2003) was administered by trained research clinicians. The SIPS is a semistructured interview measuring positive, negative, disorganized, and general symptoms. The severity of the symptoms are rated using the Scale of Prodromal Symptoms (SOPS). Each item is rated from 0 (absent) to 6 (extreme/psychotic), with higher scores indicating more prodromal symptoms.

\subsection{Procedure}

Testing was completed in a single session and lasted less than one hour (for the ToM and HiSoC). All measures were administered by trained clinicians and research assistants not blind to group status, except the HiSoC where raters were blind to group status. After complete description of the study to the participants and their legal guardians, informed consent was obtained for participants who were 18-years-old. Consent was obtained by the legal guardians for participants under 18-years-old, as well as the children's assent. Ethical approval for the study was obtained from the University of North Carolina at Chapel Hill's Institutional Review Board.

\subsection{Data analytic plan}

Statistical significance was defined as $p<.05$ and all tests were two-tailed. Chi-square tests and analyses of variance (ANOVAs) were used to examine group differences on demographic variables. Any variable that was significantly different between the groups was included as a covariate in a multivariate analysis of covariance (MANCOVA).

To evaluate the reliability and the preliminary validity of the HiSoC rating scheme, a series of psychometric analyses were performed using the entire sample $(N=54)$. Ratings were 
summed across participants on each of the factors and averaged between the scores of each pair of raters, where higher scores reflect better social functioning.

The construct validity of the HiSoC was evaluated via factor analysis. An exploratory factor analysis was conducted to examine whether the HiSoC loaded on the four proposed factors. A generalized least squares estimation (GLS) and direct oblimin rotation on the factors was employed because the item distributions did not conform to a normal distribution. The factor structure was determined by a preliminary examination of a scree plot and further investigated with a chi-square test and model fit indices.

The construct validity was further evaluated by correlating the HiSoC factors with the GF-S ratings and performance on the Eyes Test. HiSoC ratings were correlated with the SOPS subscales.

Pearson two-tailed correlations were used for all correlation analyses. The only exception was with the GF-S, where a maximum likelihood estimator of the polyserial correlation was implemented because it was non-normally distributed.

Inter-rater reliability was evaluated, where reliability was considered acceptable at Intraclass Correlations (ICCs) greater than .80. Inter-rater agreement was examined with an average, two-way random effects ICC. The internal consistency was also examined, where internal consistency was considered adequate at Cronbach's alphas greater than. 80 .

ANOVAs were conducted to examine the hypothesis that GHR adolescents would show social skill impairments compared to the NPC group (i.e., worse performance on the HiSoC). Effect sizes (Cohen's d) were calculated to determine the magnitude of differences between the groups on the HiSoC factors.

Lastly, an event trial logistic model was employed to examine the hypothesis that the GHR adolescents would show ToM deficits. An event trial logistic model accounts for the difference in the number of stimuli presented in the child and adult versions of the Eyes Test.

\section{Results}

\subsection{Group comparisons on clinical and demographic factors}

There were no significant differences between the GHR and the NPC groups on gender $\left(\chi^{2}=\right.$. $260, n s)$, ethnicity $\left(\chi^{2}=1.802, n s\right)$, age $[F(1,52)=1.605, n s]$, years of education $[F(1,52)=1.026, n s]$, or SOPS Positive symptoms $[F(1,52)=3.18, n s]$. The GHR group did, however, have significantly lower GF-S scores $[F(1,52)=13.70, p=.001]$ and more SOPS Negative $[F(1,52)=17.060, p=.000]$, Disorganized $[F(1,52)=5.62, p=.021]$, and General symptoms $[F(1,52)=10.67, p=.002]$. There was a significant difference in highest level of maternal education $\left(\chi^{2}=18.24, p=.020\right)$ between the groups with the GHR group having fewer years of maternal education. Finally, there was a trend level difference in highest level of paternal education $\left(\chi^{2}=12.76, p=.078\right)$ with the GHR group having slightly fewer years of paternal education. See Table 2 for group means and standard deviations of group clinical and demographic variables.

\subsection{Psychometric properties of the HiSoC}

3.2.1 Construct Validity-The 15 items were reduced to three factors, explaining $74 \%$ of the variance in HiSoC ratings. A three factor model fit well $\left(\chi^{2}=18.24, p=.020\right)$. There was a consensus between the scree plot and the model fit for the selection of a three factor model. Table 1 displays the factor loadings based on a generalized least squares estimation 
(GLS) and direct oblimin rotation on the three factors. The rater's impression item was not included in the factor analysis as it is a summary item.

The proposed factor structure was not fully supported by the factor analysis. The items loading onto each factor were reviewed for the common domain. As a result, the three factors identified were "Social-Interpersonal" which is composed of four items, "Affect," composed of six items, and "Odd Behavior \& Language (OB \& L)," made up of five items. Each of the HiSoC established factors was significantly positively correlated with each other, the total score and the rater's impression item (Table 3).

All three established factors (Affect, OB\& L, and Social-Interpersonal), the rater's impression item and the total HiSoC score (the three factors and rater's impression item combined) were significantly positively correlated with GF-S, such that higher GF-S ratings (i.e., better social functioning) were associated with better performance on the HiSoC (Table 4). The Social-Interpersonal factor was significantly positively correlated with the Eyes Test. The HiSoC total score, the Affect factor and the OB \& L factor were significantly negatively correlated with the SOPS Disorganized subscale. The summary item had a trend level negative correlation with the SOPS Disorganized subscale (i.e., better rater's impression scores were associated with fewer SOPS disorganized symptoms).

3.2.2 Reliability-The HiSoC demonstrated very good internal consistency for the total score (alpha $=.93$ ), as well as for each of the factors (Table 1 ). In addition, the raters reached high levels of inter-rater reliability on the established factors (ICCs: OB\& L= .90; Affect=. 92; Social-Interpersonal=.87).

\subsection{Social skill: performance on the HiSoC}

ANOVAs were conducted to examine group differences on the HiSoC (Table 5). The GHR group had significantly lower ratings on the Social-Interpersonal $[F(1,52)=13.66, p<.05]$ and Affect $[F(1,52)=5.04, p<.05]$ factors, the rater's impression item $[F(1,52)=4.76, p<.05]$ and the HiSoC total score $[F(1,52)=9.55, p<.05]$. There was a trend level difference in the OB \& $\mathrm{L}$ factor $[F(1,52)=3.06, p=.086]$, with the GHR group performing slightly worse. The effect sizes ranged from medium to large across the three factors, rater's impression item and total score.

When the above analyses were repeated using a MANCOVA to control for the SOPS subscale scores that differed between the groups (i.e., Negative, General and Disorganized subscales) and maternal level of education, the results were largely unchanged except for the rater's impression item, which was no longer statistically significant $[F(1,52)=1.27, n s]$.

\subsection{Theory of Mind}

An event trial logistic analysis showed no group differences in ToM on the Eyes Test between the GHR and NPC groups (Likelihood ratio $\chi^{2}=.93, \beta=-.11, S E=.11, n s$ ).

\section{Discussion}

The results of this study demonstrate that the HiSoC is a psychometrically reliable and valid procedure. The psychometric analyses fit a three factor model as opposed to the hypothesized four factor model. Specifically, the proposed Odd Behavior \& Language (OB\&L), Social-Interpersonal and Affect factors remained, while the proposed Communication factor was not supported. The Communication factor did not remain in the factor analysis possibly due to the fact that the initial proposed factor only contained two items. 
The HiSoC displayed preliminary construct validity as it was significantly albeit modestly correlated with GF-S scores. Although the correlations between the HiSoC factors and the GF-S were low, a strong relationship may not be expected given the GF-S is a global measure of social functioning and assesses behaviors not included in the HiSoC (e.g., quality of friendships, participation in activities, etc.). In addition, the HiSoC was significantly correlated to only the SOPS-disorganized subscale and not the positive, negative, or general subscales. This may be due to restriction of range on the SOPS as both the GHR and NPC were asymptomatic. Furthermore, other research has similarly demonstrated a relationship between increased disorganized prodromal symptoms and worse social functioning in high risk populations (Shim et al. 2008). This strengthens the HiSoC's potential to identify adolescents who demonstrate social functioning deficits and indicates that it is a statistically sound and promising measure of social skill for this population.

As hypothesized, GHR adolescents were significantly impaired on the HiSoC compared to the NPC group. Specifically, the GHR adolescents performed significantly worse on the Social-Interpersonal and Affect factors, the rater's impression item, and the HiSoC total score. There was also a trend towards worse performance on the Odd Behavior and Language factor for the GHR group. The results were generally unchanged after controlling for the SOPs subscales and maternal level of education. These findings are consistent with previous research that has found social functioning deficits in GHR adolescents using other measurement strategies, such as self-report questionnaires (Hans et al. 2000; Shim et al. 2008) and semi-structured interviews (Dworkin et al. 1994; Glatt et al. 2006).

The hypothesis that GHR adolescents would have greater impairments in ToM was not supported. The Eyes Test may be a somewhat limited ToM measure. Sabbagh (2004) discusses that ToM can be broken into two components, decoding other's mental states and reasoning about mental states. The Eyes Test measures the decoding component where visual representations are used to ascertain the mental states of the stimuli. Perhaps measures that assess the reasoning aspect of ToM, such as those that evaluate first and second-order beliefs would clarify the role of ToM as a vulnerability marker. Thus, future research examining ToM deficits in GHR adolescent samples would benefit from including a breadth of ToM measures to help clarify its role as a disease marker.

In summary, this is the first known study to evaluate a structured behavioral task of social skill in GHR adolescents. The findings highlight the role of social functioning as a potential disease marker. High risk research examining vulnerability markers, such as social functioning deficits, can improve identification of GHR adolescents at risk for psychosis and potentially inform early intervention approaches (Salokangas \& McGlashan, 2008).

\section{References}

Auther, AM.; Smith, CW.; Cornblatt, BA. Zucker-Hillside Hospital. New York: Glen Oaks; 2006. Global Functioning: Social Scale (GF: Social).

Baron-Cohen S, Wheelwright S, Hill J, Raste Y, Plumb Y. The "Reading the Mind in the Eyes" Test Revised Version: A study with normal adults and adults with asperger syndrome or high functioning autism. J. Child Psychol. Psychiatry. 2001a; 42:241-251. [PubMed: 11280420]

Baron-Cohen S, Wheelwright S, Spong A, Scahill V, Lawson J. 'Are intuitive physics and intuitive psychology independent? A test with children with asperger syndrome'. Journal of Developmental and Learning Disorders. 2001b; 5:47-78.

Brune M. "Theory of Mind" in schizophrenia: A review of the literature. Schizophr. Bull. 2005; $31: 21-42$.

Calkins ME, Curtis CE, Grove WM, Iacono WG. Multiple dimensions of schizotypy in first degree biological relatives of schizophrenia patients. Schizophr. Bull. 2004; 30:317-325. 
Corcoran, R. Theory of mind and schizophrenia, in. In: Corrigan, PW.; Penn, DL., editors. Social Cognition and Schizophrenia. Washington, DC: American Psychological Association; 2001. p. 149-174.

Couture SM, Penn DL, Roberts DL. The Functional Significance of Social Cognition in Schizophrenia: A Review. Schizophr. Bull. 2006; 32:44-63.

Docherty NM, Gordinier SW, Hall MJ, Dombrowski ME. Referential communication disturbances in the speech of nonschizophrenic siblings of schizophrenia patients. J. Abnorm. Psychol. 2004; 113:399-405. [PubMed: 15311985]

Dworkin RH, Lewis JA, Cornblatt BA, Erlenmeyer-Kimling L. Social competence deficits in adolescents at risk for schizophrenia. J. Nerv. Ment. Dis. 182:103-108.

First, MB.; Gibbon, M.; Spitzer, RL.; Williams, JBW. Biometrics Research Department. New York: New York State Psychiatric Institute; 2002. Structured Clinical Interview for DSM-IV-TR Axis I Disorders.

Glatt SJ, Stone WS, Farone SV, Seidman LJ, Tsuang MT. Psychopathology, personality traits and social development of young first-degree relatives of patients with schizophrenia. Br. J. Psychiatry. 2006; 189:337-345. [PubMed: 17012657]

Gramer M, Saria K. Effects of social anxiety and evaluative threat on cardiovascular responses to active performance situations. Biol. Psychol. 2007; 74:67-74. [PubMed: 16950557]

Hans SL, Auerbach JG, Asarnow JR, Styr B, Marcus J. Social Adjustment of Adolescents at Risk for Schizophrenia: The Jerusalem Infant Development Study. J. Am. Acad. Child. Adolesc. Psychiatry. 2000; 39:1406-1414. [PubMed: 11068896]

Irani F, Platek SM, Panyavin IS, Calkins ME, Kohler C, Siegal SJ, Schachter M, Gur RE, Gur RC. Self-face recognition and theory of mind in patients with schizophrenia and first-degree relatives. Schizophr. Res. 2006; 88:151-160. [PubMed: 16979876]

Janssen I, Krabbendam L, Jolles J, van Os J. Alterations in theory of mind in patients with schizophrenia and non-psychotic relatives. Acta Psychiatr. Scand. 2003; 108:110-117. [PubMed: 12823167]

Kaufman J, Birmaher B, Brent D, Rao U, Flynn C, Moreci P, Williamson D, Ryan N. Schedule for affective disorders and schizophrenia for school-age children present and lifetime version (KSADS-PL): initial reliability and validity data. J. Am. Acad. Child. Adolesc. Psychiatry. 1997; 36:980-987. [PubMed: 9204677]

Kelemen O, Keri S, Must A, Benedek G, Janka Z. No evidence for impaired 'theory of mind' in unaffected first-degree relatives of schizophrenia patients. Acta Psychiatr. Scand. 2004; 110:146149. [PubMed: 15233715]

Kendler KS, McGuire M, Gruenberg AM, Walsh D. Schizotypal symptoms and signs in the Roscommon Family Study. Their factor structure and familial relationship with psychotic and affective disorders. Arch. Gen. Psychiatry. 1995; 52:296-303. [PubMed: 7702446]

Kosson DS, Byrnes KA, Park S. The Interpersonal Measure of Schizoidia and Schizotypy (IM-SS). 1999 Unpublished test.

Litter J, Walker EF. Interpersonal behavior of preschizophrenic children: a study of home-movies. Child Psychiatry Hum. Dev. 1993; 23:283-295. [PubMed: 8325136]

Marjoram D, Miller P, McIntosh AM, Cunningham Owens DG, Johnstone EC, Lawrie S. A neuropsychological investigation into 'Theory of Mind' and enhanced risk of schizophrenia. Psychiatry Res. 2006; 144:29-37. [PubMed: 16904190]

Maxwell, M. Family Instrument for Genetic Studies (FIGS). Washington, D.C: Clinical Neurogenetics Branch, Intramural Research Program, National Institute of Mental Health; 1996.

McGlashan, TH.; Miller, TJ.; Woods, SW.; Rosen, JL.; Hoffman, RE.; Davidson, L. New Haven, Connecticut: PRIME Research Clinic, Yale School of Medicine; 2003. Structured Interview for Prodromal Syndromes: Version 4.0. unpublished manuscript

Mueser, KT.; Bellack, AS. Social skills and social functioning. In: Mueser, KT.; Tarrier, N., editors. Handbook of social functioning in schizophrenia. Boston: Allyn and Bacon; 1998. p. 79-98.

Roncone R, Falloon IR, Mazza M, De Risio A, Pollice R, Necozione S, Morosini P, Casacchia M. Is theory of mind in schizophrenia more strongly associated with clinical and social functioning than with neurocognitive deficits? Psychopathology. 2002; 35:280-288. [PubMed: 12457019] 
Sabbagh M. Understanding the orbitofrontal contributions to theory of mind reasoning: implications for autism. Brain. Cogn. 2004; 55:209-219. [PubMed: 15134854]

Salokangas RK, McGlashan TH. Early detection and intervention of psychosis. A review. Nordic J. Psychiatry. 2008; 62:92-105.

Schiffman J, Lam CW, Jiwatram T, Ekstrom M, Sorensen H, Mednick S. Perspective-taking deficits in people with schizophrenia spectrum disorders: A prospective investigation. Psychol. Med. 2004a; 34:1581-1586. [PubMed: 15724888]

Schiffman J, Walker E, Ekstrom M, Schulsinger F, Sorensen H, Mednick S. Childhood videotaped social and neuromotor precursors of schizophrenia: A prospective investigation. Am. J. Psychiatry. 2004b; 161:2021-2027. [PubMed: 15514402]

Shim G, Kang DH, Sun Chung Y, Young Yoo S, Young Shin N, Soo Kwon J. Social functioning deficits in young people at risk for schizophrenia. Aust. N. Z. J. Psychiatry. 2008; 42:678-685. [PubMed: 18622775]

Sprong M, Schothorst P, Vos E, Hox J, Van Engeland H. Theory of Mind in schizophrenia. Br. J. Psychiatry. 2007; 191:5-13. [PubMed: 17602119]

Stone WS, Farone SV, Seidman LJ, Olson EA, Tsuang MT. Searching for the liability to schizophrenia: Concepts and methods underlying genetic high-risk studies of adolescents. J. Child Adolesc. Psychopharmacol. 2005; 15:403-417. [PubMed: 16092907]

Tarbox S, Pogue-Geile M. Development of social functioning in preschizophrenia children and adolescents: A systematic review. Psychol. Bull. 2008; 134:561-583. [PubMed: 18605820]

Walker EF, Grimes KE, Davis DM, Smith AJ. Childhood precursors of schizophrenia: facial expressions of emotion. Am. J. Psychiatry. 1993; 150:1654-1660. [PubMed: 8214173] 


\section{Table 1}

Factor loadings $\&$ internal consistency for the established factors $(N=54)$

\begin{tabular}{cccc}
\hline Factor & Cronbach Alpha & Items & Factor loading \\
\hline Social-Inter & 0.89 & Fluency of speech & 0.96 \\
& & Guardedness & 0.78 \\
& & Social anxiety & 0.74 \\
Affect & \multirow{2}{*}{0.91} & Engagement & 0.90 \\
& & Facial affect & 0.88 \\
& & Nonverbal affect & 0.76 \\
& & Appropriate affect & 0.89 \\
& & Verbal expression & 0.88 \\
& & Gaze & 0.66 \\
OB\& L & & Anergia & 0.77 \\
& \multirow{3}{*}{0.85} & Speech valence & 0.73 \\
& & Appearance & 0.66 \\
& & Odd speech & 0.89 \\
& & Tangential speech & 0.73 \\
& & Clear communication & 0.75 \\
\hline
\end{tabular}

Note. Social Inter=Social-interpersonal; OB \& L=Odd Behavior and Language. 
Table 2

Group comparisons of clinical and demographic variables

\begin{tabular}{|c|c|c|c|c|}
\hline \multirow[t]{2}{*}{ Variable } & \multicolumn{2}{|c|}{$\begin{array}{c}\text { NPC } \\
(n=31)\end{array}$} & \multicolumn{2}{|c|}{$\begin{array}{c}\text { GHR } \\
(n=23)\end{array}$} \\
\hline & $\mathrm{N}$ & $\%$ & $\mathrm{~N}$ & $\%$ \\
\hline Female & 14 & 45 & 12 & 52 \\
\hline \multicolumn{5}{|l|}{ Ethnicity } \\
\hline $\begin{array}{l}\text { African- } \\
\text { American }\end{array}$ & 7 & 23 & 7 & 30 \\
\hline Caucasian & 22 & 71 & 16 & 70 \\
\hline \multirow[t]{2}{*}{ Asian } & 2 & 6 & 0 & 0 \\
\hline & Mean & $\mathrm{SD}$ & Mean & $\mathrm{SD}$ \\
\hline Age & 13.23 & 2.49 & 14.09 & 2.45 \\
\hline Years of Education & 6.87 & 2.40 & 7.57 & 2.61 \\
\hline GF-S $a$ & 9.16 & .58 & 8.26 & 1.18 \\
\hline SOPS-positive & 1.16 & 1.71 & 2.13 & 2.28 \\
\hline SOPS-negative $^{a}$ & .55 & 1.23 & 3.26 & 3.37 \\
\hline SOPS-disorganized $^{a}$ & .45 & .89 & 1.21 & 1.48 \\
\hline SOPS-general $^{a}$ & .32 & .70 & 1.52 & 1.88 \\
\hline
\end{tabular}

Note. NPC=Non-Psychiatric Control; GHR=Genetic High Risk; GF-S=Global Functioning Social Scale; SOPS= Scale of Prodromal Symptoms, Positive, Negative, Disorganized and General symptom subscales.

${ }^{a}$ Denotes statistically significant difference between the groups on that variable, $p<.05$. 


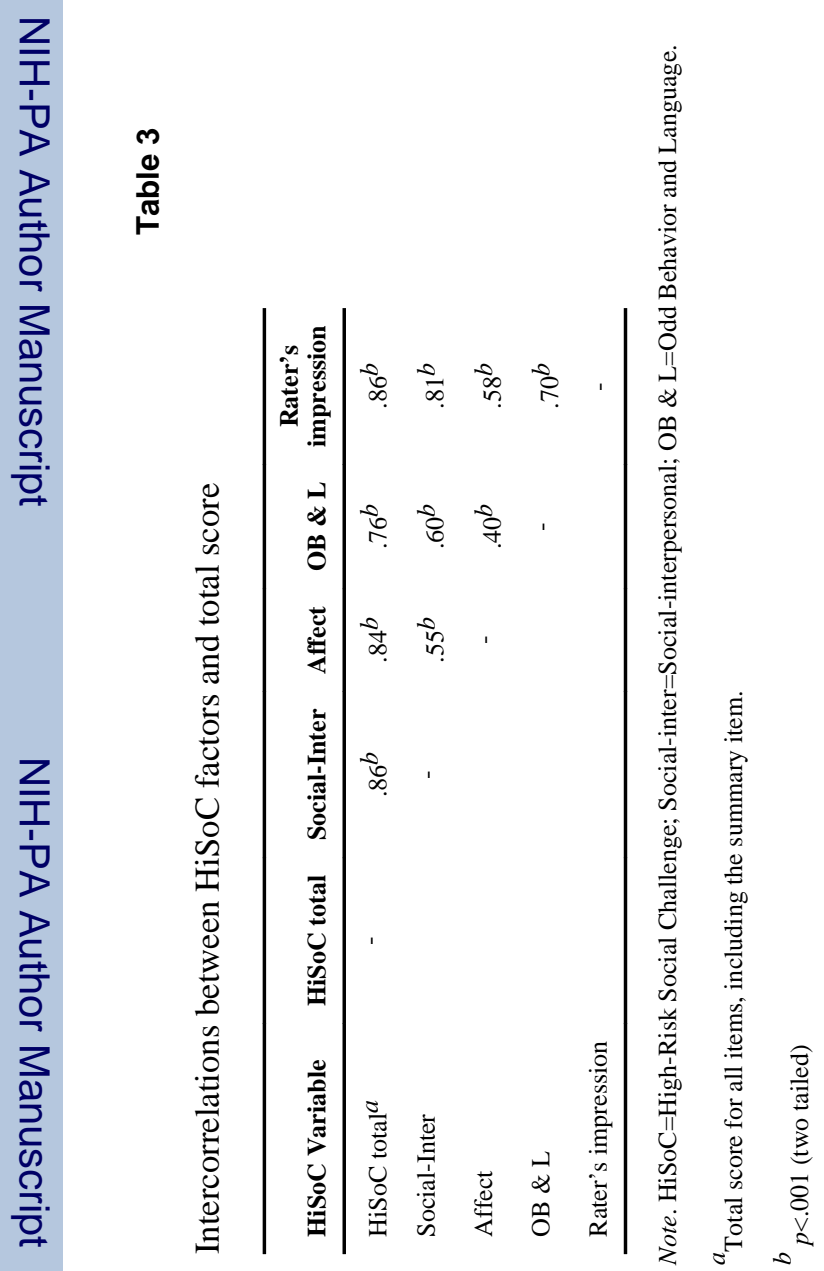




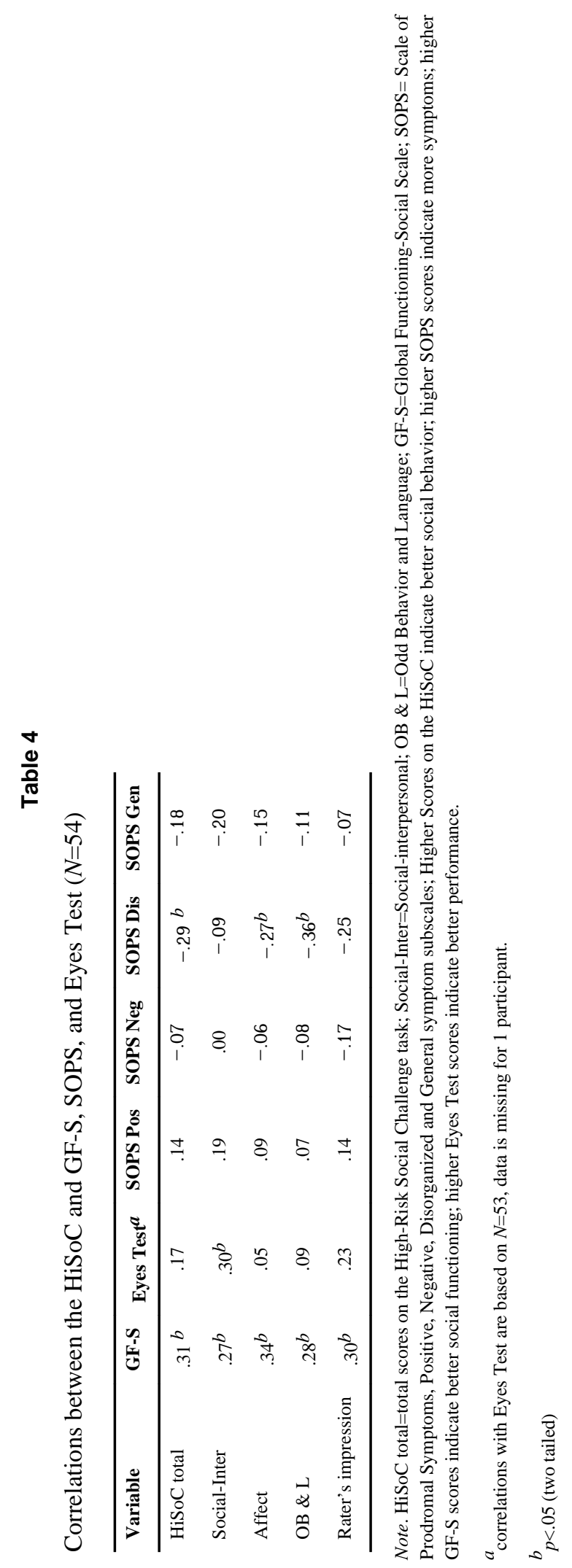

Schizophr Res. Author manuscript; available in PMC 2011 September 1. 
Table 5

\begin{tabular}{lcccc}
\hline & \multicolumn{5}{c}{$\begin{array}{c}\text { NPC } \\
(\boldsymbol{n}=\mathbf{3 1})\end{array}$} & $\begin{array}{c}\text { GHR } \\
(\boldsymbol{n}=\mathbf{2 3})\end{array}$ \\
\hline HiSoC variable & Score range $^{\boldsymbol{a}}$ & $\boldsymbol{M}(\boldsymbol{S D})$ & $\boldsymbol{M}(\boldsymbol{S D})$ & Effect size $($ Cohen's $\boldsymbol{d})$ \\
\hline Social-Inter & $4-20$ & $15.87(2.35)_{\mathrm{b}}$ & $12.35(4.56)_{\mathrm{c}}$ & 1.04 \\
Affect & $6-30$ & $23.69(4.57)_{\mathrm{b}}$ & $20.43(6.11)_{\mathrm{c}}$ & 0.63 \\
OB \& L & $5-25$ & $22.05(3.10)_{\mathrm{b}}$ & $20.41(3.76)_{\mathrm{b}}$ & 0.49 \\
Rater's impression & $1-5$ & $3.52(.94)_{\mathrm{b}}$ & $2.87(1.24)_{\mathrm{c}}$ & 0.61 \\
HiSoC total & $16-80$ & $65.13(7.33)_{\mathrm{b}}$ & $56.07(13.97)_{\mathrm{c}}$ & 0.87 \\
\hline
\end{tabular}

Group means, standard deviations, and effect sizes on the HiSoC

Note. Higher scores indicate better performance. HiSoC=High-Risk Social Challenge; Social-Inter=Social-interpersonal; OB \& L= Odd Behavior $\&$ Language Factor; NPC=Non-Psychiatric Control; GHR=Genetic High Risk; Means in the same row having different subscripts are significantly different at $p<.05$

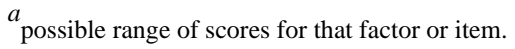

